\title{
ON THE RETENTION OF GLOBULAR CLUSTER NEUTRON STARS
}

\author{
G.A. DRUKIER \\ Institute of Astronomy, University of Cambridge \\ Madingley Rd., Cambridge CB3 OHA, UK
}

\section{Introduction}

Although globular clusters are known to contain a population of neutron stars, the recent finding by Lyne and Lorimer (1994) that field neutron stars are formed with a mean "kick" velocity of $450 \mathrm{~km} \mathrm{~s}^{-1}$ implies that globular clusters would retain very few neutron stars. The number of neutron stars is important when discussing cluster mortality and the formation rate of millisecond pulsars.

Here, I estimate the number of neutron stars retained by globular clusters assuming the that Lyne and Lorimer kick-velocity distribution is correct. Cluster evolution is followed using a direct numerical integration of the Fokker-Planck equation. I calculate the retained fraction of neutron stars as a function of kick velocity $f_{r e t}\left(v_{k}\right)$ by a Monte Carlo technique and the retained number by integrating this over the kick velocity distribution. This work is described more fully in a paper submitted to MNRAS.

\section{Results}

Scenario 1, all neutron star progenitors are single: For the most part, less than $1 \%$ are retained, only the models with mass $5 \times 10^{6} M_{\odot}$ retain more than $3 \%$. The fraction retained increases with the depth of the cluster potential.

Scenario 2, all neutron star progenitors are massive binaries: The results under this scenario are based on those of Brandt and Podsiadlowski (1995) (especially §3.2) who estimated the effects of the Lyne and Lorimer kick velocities on the evolution of massive binaries. For each of the three possible 


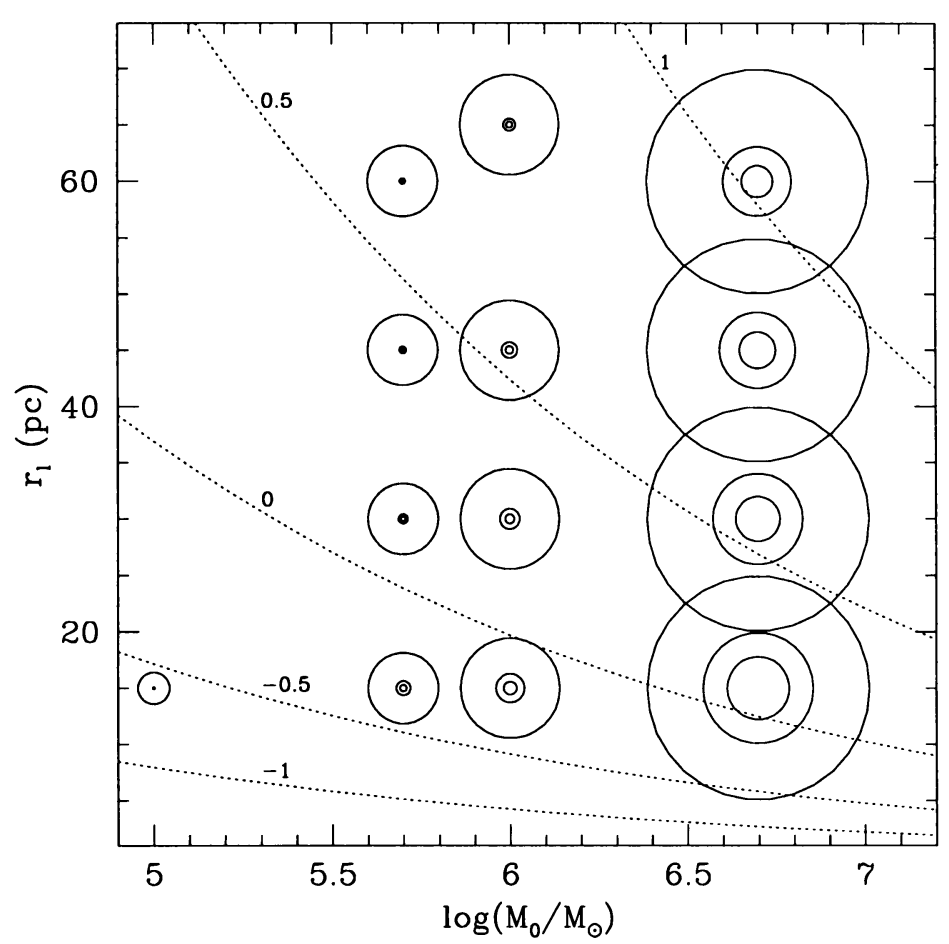

Figure 1. For $W_{0}=7$ and $x=1$, the figure shows the number of neutron star progenitors and retained neutron stars under the two scenarios as a function of $M$ and $r_{l}$. The areas of the circles are proportional to the number of stars. For each model, the largest circle gives the number of massive stars initially in the cluster. The smallest circle gives the number of neutron stars retained under the single-star scenario and the medium circle gives the number under the binary scenario. The dotted curves are curves of constant half-mass relaxation time as labelled. The units are log Gyr.

outcomes after the first supernova ( 2 unbound stars, binary with new orbit, or merged system) the velocity distributions of the resulting stars or systems were combined with $f_{r e t}\left(v_{k}\right)$ to give the retained numbers. Most of the retained neutron stars come from the $\sim \frac{1}{4}$ of systems which remain bound. It can be argued that most of these systems will pass through a high-mass $\mathrm{x}$-ray binary phase leaving a single neutron star in the cluster. The number retained under this scenario is generally 2 to 5 times larger than the number retained in the first scenario.

\section{References}

Brandt N., Podsiadlowski P. 1995, MNRAS, 274, 461

Lyne A. G., Lorimer D. R. 1994, Nature, 369, 127 